\title{
Design of Elastomer Structure to Facilitate Incorporation of Expanded Graphite in Silicones Without Compromising Electromechanical Integrity
}

\author{
Hassouneh, Suzan Sager; Daugaard, Anders Egede; Skov, Anne Ladegaard
}

Published in:

Macromolecular Materials \& Engineering

Link to article, DOI:

10.1002/mame.201400383

Publication date:

2015

Document Version

Peer reviewed version

Link back to DTU Orbit

Citation (APA):

Hassouneh, S. S., Daugaard, A. E., \& Skov, A. L. (2015). Design of Elastomer Structure to Facilitate Incorporation of Expanded Graphite in Silicones Without Compromising Electromechanical Integrity. Macromolecular Materials \& Engineering, 300, 542-550. https://doi.org/10.1002/mame.201400383

\section{General rights}

Copyright and moral rights for the publications made accessible in the public portal are retained by the authors and/or other copyright owners and it is a condition of accessing publications that users recognise and abide by the legal requirements associated with these rights.

- Users may download and print one copy of any publication from the public portal for the purpose of private study or research.

- You may not further distribute the material or use it for any profit-making activity or commercial gain

- You may freely distribute the URL identifying the publication in the public portal 
DOI: 10.1002/mame.((insert number)) ((or ppap., mabi., macp., mame., mren., mats.))

\section{Full paper}

Design of Elastomer Structure to Facilitate Incorporation of Expanded Graphite in Silicones without Compromising Electromechanical Integrity

Suzan Sager Hassouneh, Anders Egede Daugaard, Anne Ladegaard Skov*

S. S. Hassouneh, Dr. A.E. Daugaard, Dr. A.L. Skov

The Technical University of Denmark, Søltofts Plads build. 227, 2800 Kgs. Lyngby, Denmark E-mail: al@kt.dtu.dk 
The development of elastomer materials with a high dielectric permittivity has attracted increased interest over the last years due to their use in for example dielectric elastomers. For this particular use, both the electrically insulating properties - as well as the mechanical properties of the elastomer - have to be tightly controlled in order not to destroy favorable elastic properties by the addition of particles. This study focuses on improving the electromechanical properties of an enhanced PDMS matrix with expanded graphite (EG) as filler. The PDMS matrix is crosslinked by means of an 8-functional crosslinker, which allows for development of a suitable network matrix. The dielectric permittivity was increased by almost a factor of 4 compared to a benchmark silicone elastomer.

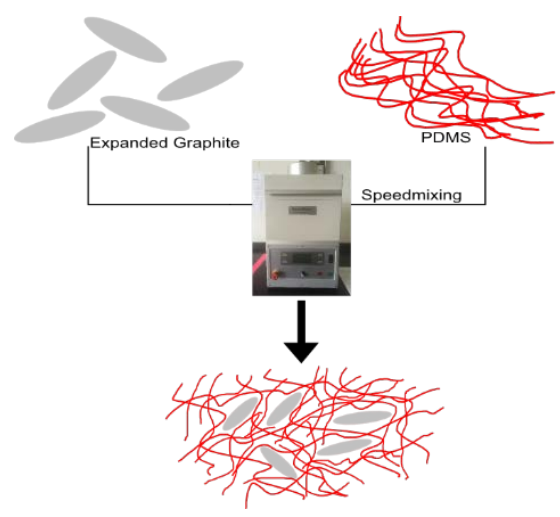

FIGURE FOR ToC_ABSTRACT

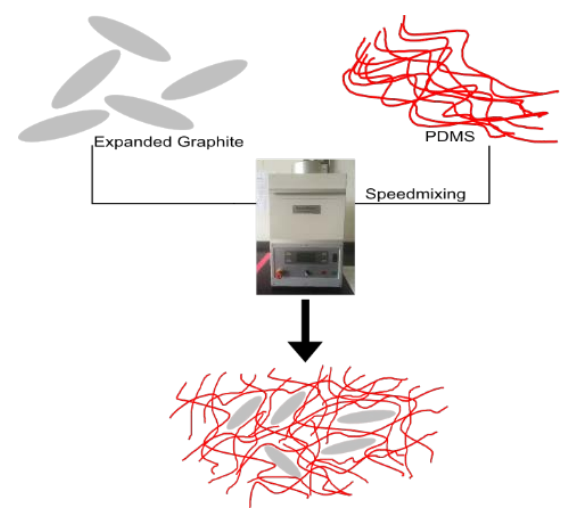

The structure of silicone network was modified to facilitate better incorporation of large sized conducting fillers for better dispersion and better mechanical and electrical properties. 


\section{Introduction}

Utilization of DE has increased with novel applications, amongst many applications soft lenses $^{[1]}$ and photonic crystal elastomers ${ }^{[2]}$ can be mentioned. Energy harvesting from waves have also attracted major attention. ${ }^{[3]}$ However most studies are still based on un-optimized elastomers and for commercial products to enter the market, increased energy densities of the elastomers are vital. Some of the advantages of using DEs compared to other traditional transducer systems are the potential low cost, low weight, high elasticity, fast response and large strains (more than $300 \%) .{ }^{[4]}$ So for further optimization it is important not to compromise the favorable properties significantly.

DEs consist of an elastomer film sandwiched between two compliant electrodes. A voltage is applied over the electrodes and the elastomer responds by deforming due to the electrostatic forces between the compliant electrodes. ${ }^{[5]}$ The elastomer therefore contracts in thickness and expand in area. In a given electrical field (E) the mechanical response of the elastomer can be expressed by

$-p_{\text {Maxwell }}=\frac{\varepsilon_{0} \cdot \varepsilon_{r} \cdot E^{2}}{Y}=\frac{\varepsilon_{0} \cdot \varepsilon_{r}}{Y} \cdot\left(\frac{U}{d}\right)^{2}$

where $\mathrm{p}$ is the strain, $\varepsilon_{0}$ is the vacuum permittivity, $\varepsilon_{\mathrm{r}}$ is the relative dielectric constant, $\mathrm{Y}$ is the Young's modulus, $\mathrm{U}$ is the voltage and $\mathrm{d}$ is the original thickness of material. ${ }^{[6]}$

The above equation takes into account only the strain induced by the electrostatic field (Maxwell effect) but also the electrostriction needs to be accounted for ${ }^{[7,8]}$

$$
-p_{\text {electrostriction }}=Q \varepsilon_{0}{ }^{2}\left(\varepsilon_{r}-1\right)^{2}\left(\frac{U}{d}\right)^{2}
$$

where Q is the electrostrictive coefficient. The overall strain can then be written

$$
p_{\text {tot }}=p_{\text {Maxwell }}+p_{\text {electrostriction }}=M \cdot\left(\frac{U}{d}\right)^{2}
$$


where $\mathrm{M}$ is the apparent electrostrictive coefficient.

One way to increase the achievable strain is by increasing the working voltage, which is not desirable, since the applicability of the DE elements will be limited due to the very expensive electronics required. Another parameter that can be changed and has a great impact on the strain (as seen from equation 1) is the thickness of the elastomer. However, for many systems the thickness of the films is already reduced so much that it may cause processing challenges to reduce it further. ${ }^{[9]}$ Therefore, to improve the actuation performance without changing the applied voltage and thickness, the most common approach is to increase the dielectric permittivity of the elastomer without increasing the Young's modulus to the same extent.

The mechanical and electrical losses of the material have an influence on the overall performance of a transducer. The losses indicate the dissipative nature of the material, i.e. energy lost to heating. ${ }^{[10]}$ Therefore the losses should be minimized such that most of the applied energy is converted into mechanical energy in actuation mode and into electrical energy in generator mode. While the mechanical losses are due to viscoelasticity, the electrical losses arise from leakage currents across the film, build-up of charges and resistive losses in the electrodes. ${ }^{[11]}$

The addition of conductive fillers in the matrix influences the dielectric losses. If the fillers are not well-dispersed they tend to agglomerate and form conductive paths and hence increase the dielectric losses. ${ }^{[12]}$ Additionally the addition of hard fillers to soft elastomers changes the mechanical properties of the resulting composite. When the filler is added above its percolation threshold the hard fillers form a continuous, rigid structure in the elastomer and are thus very reinforcing. Also the interaction between filler and elastomer will strongly influence the properties of the composite. ${ }^{[13]}$

Traditionally, the research on elastomers for DE has focused on acrylics and polydimethylsiloxane (PDMS). ${ }^{[4]}$ Especially PDMS has shown promising features. PDMS elastomers have favorable properties, such as a low glass transition temperature $\left(\mathrm{T}_{\mathrm{g}}=\right.$ - 
$124{ }^{\circ} \mathrm{C}$ ) and a good resistance to environmental degradation like oxygen, ozone and sunlight. ${ }^{[14]}$ Another advantage of using PDMS is the possibility of tailoring the elastomer to achieve the desired elastic properties. ${ }^{[15,16]}$ This is possible by controlling the stoichiometric ratio (usually denoted r) of the reactive groups and the crosslinker. For the optimization of silicone elastomers with respect to the dielectric properties, two main approaches exist namely modification of the polymers in the network ${ }^{[17-20]}$ and addition of high permittivity fillers. ${ }^{[4,21,22]}$ The modification of the polymer backbone is costly due to the additional synthesis steps but this technique causes the least destruction of the mechanical properties of the elastomers, which can be seen from swelling experiments. ${ }^{[18-20]}$ Addition of conducting particles adds only slightly to the price of the resulting elastomer but does on the other hand usually introduce large changes in the mechanical properties of the resulting elastomer due to the combination of hard fillers and soft polymer network. As a new type of high-permittivity elastomers interpenetrating networks of covalent silicone and ionic components have been investigated. ${ }^{[22,23]}$

Fillers can be added and blended in a facile way, since the crosslinking reaction can be delayed by adding an inhibitor, which allows time for dispersion of fillers. Different types of fillers have been investigated to reinforce the properties of the PDMS elastomers. Conductive fillers such as Single Walled Carbon Nanotubes, ${ }^{[24]}$ Multi Walled Carbon Nanotubes, ${ }^{[25]}$ graphite and carbon black ${ }^{[26,27]}$ have shown enhancement in the electrical and mechanical properties. One disadvantage of adding these fillers is the increase in stiffness with increased loadings. Higher loadings of the filler in the system also result in increased tendency of electrical breakdown due to electrical percolation. For these reasons, it is not favorable to have high concentrations of conductive fillers in the system. As discussed by Stoyanov et $a l^{[28]}$ subpercolative systems have the disadvantage of showing a sample thickness dependent percolation threshold far above from what is expected. For their system consisting of carbon 
black in a thermoplastic copolymer they estimated bulk properties to appear above $700 \mu \mathrm{m}$, which is far above the usual thicknesses of DE films $(\sim 10-100 \mu \mathrm{m})$.

An alternative to the previous mentioned fillers is expanded graphite, since it is not expected to increase the stiffness substantially. ${ }^{[29]}$ Natural graphite consists of multiple sheets of graphene held together by van der Waals forces, as shown in Figure 1a. By introducing the

graphite to small molecules e.g. acids ${ }^{[30,31]}$ between the graphene layers followed by a rapid heating the van der Waals bonds are broken, the acid is vaporized and the graphene sheets separates (Figure 1b). This type of graphite can be dispersed into the polymer matrix and provide an exfoliated graphite composite as sketched in Figure 1c.

\section{((Figure 1 Insert))}

The dispersion of expanded graphite into the polymer matrix is not trouble-free. The expanded graphite has a tendency to form agglomerates despite the larger distance between the graphene layers. These agglomerates have a negative impact on the electrical and mechanical properties. The polymer matrix has better compliance with the exfoliated graphite sheets than the agglomerates. A recent study was focused on dispersion methods for different loadings of EG in a 4-functional crosslinked networks. ${ }^{[21]}$ The study showed that speed mixing the samples gave the best dispersion of the EG fillers in the matrix and the percolation threshold was reached at loading of 5 wt\% of EG. ${ }^{[21]}$ Upon stretching of these composites it was obvious that voids were introduced around the interfaces of EG and silicone. This indicates that the network is too weak locally around the EG and thus needs reinforcement which can be introduced by increasing the functionality of the crosslinker. ${ }^{[32]}$ The purpose of this study is to facilitate successful introduction of EG into silicones without compromising the elasticity of the elastomer by adjustment of the polymer network. An 8-functional crosslinker is applied to form networks that are loaded with various concentrations of EG (2-4 
$w t \%)$ to investigate the electromechanical properties of more compliant networks with less voids and thus better electromechanical properties.

\section{Experimental Section}

\subsection{Materials and Equipment}

The samples are prepared by use of PDMS (Polydimethylsiloxane - DMS-V35 - 49500 g/mol) and 8-functional crosslinker (Methylhydrosiloxane-dimethylsiloxane copolymerHMS-301- 1900-2000 g/mol), both purchased from Gelest Inc. The inhibitor (Pt88) and the platinum catalyst (platinum cyclovinylmethyl-siloxane complex, 511) were provided by Hanse Chemie AG. The graphite used was commercially available expanded graphite TIMREX ${ }^{\circledR}$ BNB90 supplied by Timcal. The size distribution of the particles was reported by the supplier as $50 \%$ of the graphite below $36 \mu \mathrm{m}$ and $90 \%$ below $85 \mu \mathrm{m}$, with a specific surface area of $28.4 \mathrm{~m}^{2} / \mathrm{g}$. The benchmark silicone elastomer is commercially available Elastosil RT625 purchased from Wacker Chemie AG.

For preparation of the samples a Speedmixer ${ }^{\mathrm{TM}}$ is used. The Speedmixer ${ }^{\mathrm{TM}}$ from Flack Tek Inc. is a Dual Asymmetric Centrifuge (DAC) 10 FVZ-K, which operates in the interval from 300$3500 \mathrm{rpm}$. The preparation of thin films was performed on an Elcometer 4340 automatic film applicator with a gap of $200 \mu \mathrm{m}$ leading to film thicknesses of $\sim 120-150 \mu \mathrm{m}$. The rheological measurements were carried out on a controlled stress rheometer (AR2000) from TA Instruments and the samples had a diameter of $25 \mathrm{~mm}$ and a thickness of $1 \mathrm{~mm}$.

The dielectric permittivity measurements were carried out on the ARES-G2 rheometer with the Dielectric Thermal Analysis accessory from TA Instruments. The diameter of electrodes was $25 \mathrm{~mm}$.

An in-house-built device was used for measuring the actuation of the samples. A sample (60 $\mathrm{mm} * 20 \mathrm{~mm}$ ) was fixed on frames and an area of $20 \mathrm{~mm} * 20 \mathrm{~mm}$ was smeared with carbon grease. Conductive electrode tape was attached to the sample, in contact with the carbon 
grease, to make contact to the high voltage AC amplifier. The sample is mounted with the clamps on a moveable stage controlled by a c-863 DC motor. The top clamp has a $1 \mathrm{~kg}$ load cell attached to measure the force the film exert when actuated at different applied voltages. The measurements were carried out at various applied voltages in the interval $1-5 \mathrm{kV}$ and the samples were subjected to a $10 \%$ prestretch. Samples were actuated until electromechanical breakdown.

To measure the electrical breakdown strength an in-house-built device based on international standards (IEC 60243-1 (1998) and IEC 60243-2 (2001)) was used. The sample thicknesses were measured with a digital thickness gage (Diesella A/S, Denmark). The sample was placed between two spherical electrodes, where the electrodes were set accordingly with a micrometer stage and gauge. To ensure good contact between the sample and the electrodes and indent of less than 5\% was added and the breakdown strength was measured at the point of contact. The sample is measures at 12 points and an average of the measurements are indicated as the breakdown strength of the sample.

The electrical breakdown strength was then measured with an increase in the applied voltage (50-100 V/step) at a rate of $0.5-1$ steps/s. ${ }^{[32]}$

\section{2. $\quad$ Mixing Procedure}

The process of preparing the samples was simplified by making premixes. Premix A contained vinyl terminated PDMS (DMS-V35, 49.67 g, 2 mmol end groups) and 8-functional crosslinker (Methylhydrosiloxane-dimethylsiloxane copolymer- HMS-301, 1.72 g, 6 mmol reactive groups) and Premix B contained the vinyl-terminated PDMS (DMS-V35, 50 g, 2.02 mmol end groups) and the platinum catalyst (platinum cyclovinylmethyl siloxane complex (511), $2.04 \mathrm{mg}$ ). The inhibitor was added to Premix A to allow sufficient time for thorough mixing as well as coating without significant onset of crosslinking. 
Premix A and B were combined in a Speed Mixer cup and the expanded graphite was added in various concentrations (2-4 g depending on the desired concentration (wt\%)). The samples were mixed for 5 minutes at $2750 \mathrm{rpm}$. The sample was then poured in a mold or coated on a release liner and cured in the oven for 14 hours at $80{ }^{\circ} \mathrm{C}$. Samples were prepared with different concentrations of expanded graphite (2, 3 and $4 \mathrm{wt} \%$ ) in $1 \mathrm{~mm}$ and $200 \mu \mathrm{m}$ thick films.

\section{Results and Discussion}

PDMS samples were prepared with different concentrations of expanded graphite by speed mixing samples to obtain well-dispersed samples. To ensure a good mixing of the catalyst and crosslinker into the PDMS precursor, premixes are prepared, which simplify the process as well as allowing for storage of premixes. ${ }^{[33]}$ It was previously shown that speed mixing was an efficient way to disperse EG in silicones. ${ }^{[21]}$

\subsection{Morphological Analysis}

The morphology of cured samples from mechanically mixing and speed mixing, respectively, is shown in Figure 2.

((Figure 2 Insert))

The mechanically mixed elastomer contains big agglomerates of the EG fillers. The mechanical mixing is not sufficient to break the agglomerates and thereby get a good microscale dispersion of the fillers in the PDMS matrix. By speed mixing the sample the agglomerates are broken to a larger extent and the EG fillers are better dispersed throughout the sample as illustrated in Figure 2B. The particle size varies strongly for the mechanically 
mixed samples, where agglomerates of length $\sim 200 \mu \mathrm{m}$ are rather common, to the speed mixed samples where the largest agglomerates are of the order of $50 \mu \mathrm{m}$.

\subsection{Mechanical Properties}

The mechanical properties for the samples with different loadings and the benchmark sample (Elastosil RT625) are compared and illustrated in Figure 3. Small amplitude oscillatory shear is used to investigate the mechanical properties and the fillers influence on the network.

((Figure 3 Insert))

Figure 3 shows that the storage moduli decrease with increasing loading of the EG fillers. The storage moduli for the 2 and $3 \%$ loaded samples are comparable with the benchmark silicone elastomer Elastosil RT625 within $+/-10 \%$ which is within common uncertainty on the determination of $\mathrm{G}^{\prime}$ for soft elastomers. The sample with a concentration of $4 \%$ has a significantly lower storage modulus ( a factor of 2 ). This is not what is commonly encountered in composites of thermoplastic polymers which usually show increases in G' with loading. Seen from the perspective of the actuation equation (Equation1) the decreased storage modulus is favorable, since $Y=3 G^{\prime}(\omega->0)$ and thus increased actuation should be obtained solely from the decrease in G'. However, this behavior indicates that major network destruction is taking place.

The increased interfacial area with increased loading could impose a steric hindrance and a reduction of mobility in the matrix, which is well known for nano-composites. This would result in a slower or incomplete reaction and especially affect systems with an already reduced volume as in a thin film system. In a similar manner, the increased surface area of the filler in the well dispersed reaction media, could interact with the platinum catalyst and inhibit the reaction, which again would result in a slower or incomplete reaction. The observed 
reduction in moduli with improved dispersion of the filler could also be explained based on the steric hindrance around the particles, which would result in local internal 2 dimensional (2D) curing. 2D curing is in contrast to traditional 3D curing of the elastomer in the bulk. At the surface, the dynamics of the crosslinker is strongly reduced since the crosslinker relies on self-diffusion rather than on reptation. ${ }^{[34]}$ Furthermore, the number of reactive sites surrounding the interface crosslinker is reduced by approximately a factor of 2 and thus the crosslinker becomes more susceptible to participating in loop formations or simply not reacting. Both phenomena ultimately lead to the crosslinker becoming a chain extender and thus decrease the effective crosslinking concentration. For the 4-functional crosslinker the formation of one loop causes the loss of crosslinking capability as well as two unreacted sites have the same effect. For the 8-functional crosslinker 3 times as many occurrences are required.

This surface phenomenon is very often observed at a macroscopic level for Pt-catalyzed silylation room temperature vulcanized silicone (RTV), where the surface of a sample may feel sticky or greasy whereas the bulk is completely nonsticky and fully cured. If this phenomenon is taking place, it would be expected that increased dispersion of the filler would result in a reduction in storage moduli of the samples and formation of small voids in the system.

In a previous study a 4-functional crosslinker was used. ${ }^{[21]}$ Upon comparison of the 8functional system with the previous studied 4-functional crosslinked system it can be seen that the storage moduli are lower for the 8-functional crosslinked system with the 3\% and 4\% EG loaded samples and higher for the 2\% EG loaded sample. This behavior can be explained by better incorporation of the 3\% and 4\% EG in the 8-functional network compared to the 4functional network. For the $2 \%$ loaded samples, dispersion is good in both types of networks and the 8-functional network thus has the highest elastic modulus as predicted by classical network theories. 
Table 1 shows the storage and loss moduli at $0.01 \mathrm{~Hz}$ and the viscous loss of each network.

((Table 1 Insert))

The greatest viscous loss can be seen for the 3\% EG loaded sample, while 2 and $4 \%$ have similar viscous losses as that of Elastosil RT625. This behavior can be explained by higher tendency to 2D curing and thus decreased G' with loading.

In an earlier study, ${ }^{[21]}$ a 4-functional crosslinker was used for crosslinking of the matrix. The study showed that the relative viscous dissipation between 100 and $0.01 \mathrm{~Hz}$ was of the order of 10 higher than what is reported here. This is a result of 2D curing and the occurrence of voids in the matrix. By using an 8-functional crosslinker this phenomenon is eliminated and the matrix is improved.

\subsection{Dielectric Properties}

The dielectric permittivity is measured for all DMS-V35 samples loaded with EG (2-4 wt\%) and the pure Elastosil RT625 on the ARES G2 with dielectric thermal analysis fixture. Figure 4 illustrates the dielectric measurements for the EG loaded samples with different loadings of EG and RT625 (0 wt\% EG).

((Figure 4 Insert))

The figure shows that by increasing the loading of EG the permittivity increases as expected. The permittivity for the 2 and 3 wt\% loaded samples and RT625 are almost constant over the whole frequency range while the permittivity for the $4 \mathrm{wt} \%$ loaded sample is decreasing with increasing frequency, showing a strong tendency to Maxwell polarization at low frequencies. In a previous study, ${ }^{[21]}$ where the PDMS matrix was vulcanized with a 4-functional crosslinker, the permittivity was measured to 8.6 for the $4 \%$ EG loaded sample. This increase in permittivity was caused by the large surface area of the particles being active. For the 8- 
functional crosslinked system the dielectric permittivity was measured to 12 , which is a $40 \%$ increase to the 4-functional crosslinked system and a $375 \%$ increase to the commercially available system Elastosil RT625. This increase in the dielectric permittivity could be caused by the improved matrix facilitating better distribution as well as the crosslinking density will determine the mobility in the network.

The electrical breakdown strengths are measured for the different loadings and compared to the benchmark silicone elastomer, which are depicted in Figure 5. The error bars for each sample measurement are shown as vertical lines.

((Figure 5 Insert))

The figure shows an increase in the breakdown strength with increasing loadings of EG. The breakdown strength was $40 \mathrm{~V} / \mu \mathrm{m}, 41 \mathrm{~V} / \mu \mathrm{m}$ and $48 \mathrm{~V} / \mu \mathrm{m}$ for the $2 \% \mathrm{EG}, 3 \% \mathrm{EG}$ and $4 \% \mathrm{EG}$ loaded samples, respectively. However, in comparison with the benchmark the breakdown strength $(81 \mathrm{~V} / \mu \mathrm{m})$ decreases with a factor 2 . This decrease is due to a combination of increased conductivity, changed morphologies (the benchmark material contains nonconductive silica fillers which reinforce the material) and mechanical properties when EG is added to the otherwise homogeneous silicone elastomer.

The standard deviation on the reference film is $+/-7 \mathrm{~V} / \mu \mathrm{m}$ which is rather large but not uncommon when several breakdown phenomena are occurring simultaneously. ${ }^{[35]}$ For the elastomers with EG the standard deviations drop to $+/-2 \mathrm{~V} / \mu \mathrm{m}$ which indicates that there is one single phenomenon causing the electrical breakdown. The samples with unoptimized network showed strong dependence on the location of the electrode when testing for breakdown strength such that the variation in the determination of the breakdown strength was very large with data scattered in between 20 and $50 \mathrm{~V} / \mu \mathrm{m}$ indicating the very nonhomogeneous distribution of EG. These samples were therefore not suitable candidates for 
dielectric elastomers as they would fail instanteneously when the entire elastomer was subjected to an electrical field rather than just single points.

\subsection{Electromechanical Properties}

The actuation performance of the materials with different loadings of EG was measured on 120-150 $\mu \mathrm{m}$ thick films. The thicknesses were chosen to be above the largest particles size as to avoid single-particle short-circuiting. Different voltages (1-5kV) were applied to $10 \%$ prestretched samples and the force was measured. The prestretch was introduced to soften the elastomer slightly as well as to avoid uncertainty on the zero-stress state of the elastomer and thus reduce measurement uncertainties due to wrinkling etc.

The electric field as a function of the strain is illustrated in Figure 6. The figure shows, as expected that the strain increases slightly faster for 3\% EG with increasing electric field. For the $2 \%$ sample there is good agreement between the results from the electrical breakdown measurements (Figure 5) whereas for the 3\% sample the coupled electromechanical breakdown strength is lowered with approximately $8 \mathrm{~V} / \mu \mathrm{m}$, which indicates that the electrical breakdown strength decreases with alignment of the EG plates.

((Figure 6 Insert))

The measured actuation performance is compared with the predicted actuation, which is illustrated in Figure 7. The predicted actuation performance is calculated from the measured dielectric permittivity and Young's modulus using Equation (1). Figure 6 shows that the actual measured strains are not consistent with the predicted measurements. This can be attributed to heating arising from both electrical and mechanical losses leading to an increased leakage current before the electrical field becomes so large that it exceeds the leakage. A recent study of Zakaria et al ${ }^{[36]}$ showed that even changes of $10^{\circ} \mathrm{C}$ can potentially lead to detrimental behavior of dielectric elastomers. Furthermore air voids may be introduced upon stretching leading to lower actuation. The apparent electrostrictive coefficient, $\mathrm{M}$, has been 
determined but since there seems to be no actuation of the elastomer composites before a certain threshold value, the values were not predictive.

((Figure 7 Insert))

This difference between the predicted and measured actuation at higher electric field are approximately a factor of 5 for the 2\% EG loaded samples and approximately 30 for the 3\% EG loaded sample. The difference in the measured and predicted values can be caused by a leakage current, which indicates that the material is not a perfect insulator and has some conductivity. Breakdown may also be facilitated by voids at the interfaces upon stretch where the local deformation may be anisotropic due weak interfacial forces between EG sheets and silicone matrix.

The dimension of the EG sheets are of the order of $60 \mu \mathrm{m}$ (as determined from the optical images in Figure 2B). For comparison the end-to-end distance of the polymer applied in this study is calculated. ${ }^{[3]}$ It is assumed that the PDMS polymer chain has an average molecular weight $\mathrm{M}=50 \mathrm{~kg} / \mathrm{mol}$, the average bond length is $1.5 \AA$ and that the polymer chain can be described by a simple random walk with step length of the repeat unit [-Si-(CH3)2-O] $\left(\mathrm{M}_{\mathrm{o}}\right.$ $=74 \mathrm{~g} / \mathrm{mol}$ ). The number of repeat units is $\mathrm{N}=\mathrm{M} / \mathrm{M}_{\mathrm{o}}=50103 / 74=677$ and the length of one repeat unit consisting of two bonds is $b=2 * 1.5 \AA=3 \AA$. The (random walk) end-to-end distance is $\mathrm{R}=\sqrt{\mathrm{N}} * \mathrm{~b}=\sqrt{677} * 3 \AA=78 \AA$. The area of an EG plate is assumed to be $60 \mu \mathrm{m}$ times $60 \mu \mathrm{m}$. From these calculations it is obvious that the 2D effect is rather significant, since the area of one EG facilitates approximately $6 * 10^{7} 2 \mathrm{D}$ crosslinks. This large number indicates the significance of the local environment of the interface between elastomer and EG. Therefore it is important that the crosslinker provides the possibility of forming a $2 \mathrm{D}$ network at the surface with similar stress releasing properties as the matrix such that stress-induced voids are minimized. The 8-functional crosslinker facilitates a stronger network in the surface since the 
crosslinker does not need to be fully reacted in order to act as a crosslinker whereas for the 4functional crosslinker is acting as chain extender if two of the reactive sites are participating in reaction with the same end-functionalized PDMS, i.e. a loop, or if the local environment allows for two reactions only.

\section{Conclusion}

The silicone matrix is attempted improved by using an 8-functional crosslinker instead of a

previous studied 4-functional crosslinked system. ${ }^{[21]}$ The samples are loaded with different concentrations (2-4 wt\%) of EG to investigate the influence of EG on the electromechanical properties.

The mechanical properties were investigated and showed that the material got softer with increasing loadings of EG. This is explained by the two-dimensional curing taking place at the interface between silicone and EG. In contrast to the previously reported 4-functional crosslinker, the 8-functional crosslinker facilitated better dispersion as well as the electromechanical properties were improved since the interfaces were better crosslinked and the void formation upon stretching was reduced.

It was shown that the dielectric permittivity was increased with increasing loadings of EG. The dielectric permittivity for the 4\% EG 8-functional crosslinked system was increased with $40 \%$ to a value of 12 compared to the $4 \%$ EG loaded 4-functional crosslinked system and a 375\% increase compared to Elastosil RT625.

The electrical breakdown strength increases with increasing loadings of EG, where the breakdown strength was $40 \mathrm{~V} / \mu \mathrm{m}$ for the $2 \% \mathrm{EG}, 41 \mathrm{~V} / \mu \mathrm{m}$ for the $3 \% \mathrm{EG}$ and $48 \mathrm{~V} / \mu \mathrm{m}$ for the 4\% EG sample. Compared to the benchmark the breakdown strength decreases with a factor 2 but this was expected since the benchmark is filled with non-conductive silica particles. 
The predicted actuation was calculated from the measured dielectric permittivity and Young's modulus and compared with the measured actuation. The measured actuation was approximately a factor 5 lower than the predicted measurements for the 2\% EG loaded samples and a factor of approximately 30 for the 3\% EG loaded sample.

\section{Appendix/Nomenclature/Abbreviations}

Acknowledgements: The authors would like to thank the Danish Agency for Science and InnovationsFonden for financial support. Sindhu Vudayagiri is acknowledged for her help with the actuation measurements.

Received: Month XX, XXXX; Revised: Month XX, XXXX; Published online:

((For PPP, use “Accepted: Month XX, XXXX” instead of "Published online”)); DOI: 10.1002/marc.((insert number)) ((or ppap., mabi., macp., mame., mren., mats.))

Keywords: PDMS, expanded graphite, electrical properties, capacitance

[1] F. Carpi, G. Frediani, S. Turco, D. De Rossi, Adv. Funct. Mater. 2011, 21, 4152.

[2] Q. Zhao A. Haines, D. Snoswell, C. Keplinger, R. Kaltseis, S. Bauer, I. Graz, R. Denk, P. Spahn, G. Hellmann, J. J. Baumberg, Appl. Phys. Lett. 2012, 100, 101902.

[3] S. J. A. Koh, C. Keplinger, T. Li, S. Bauer, Z. Suo, IEEE/ASME Trans. Mechatronics 2011, 16, 33.

[4] P. Brochu, Q. Pei, Macromol. Rapid Commun. 2010, 31, 10.

[5] X. Zhang, C. Löwe, M. Wissler, B. Jähne, G. Kovacs, Adv. Eng. Mater. 2005, 7, 361.

[6] R. Pelrine, R. Kornbluh, Q. Pei, J. Joseph, Science 2000, 287, 836. 
[7] Z. Suo, X. Zhao, W. Greene, J. Mech. Phys. Solids 2008, 56, 467.

[8] B. Guiffard, L. Seveyrat, G. Sebald, D. Guyomar, J. Phys. D. Appl. Phys. 2006, 39, 3053.

[9] S. Vudayagiri, M. D. Junker, A. L. Skov, Polym. J. 2013, 45, 871.

[10] R. Kornbluh, R. Pelrine, Q. Pei, S. Oh, J. Joseph, Proc. SPIE 2000, 3987.

[11] R. D. Kornbluh R. Pelrine, H. Prahlad, A. Wong-Foy, B. McCoy, S. Kim, J. Eckerle, and T. Low, Proc. SPIE 2011, 7976, 797605.

[12] M. Molberg D. Crespy, P. Rupper, F. Nüesch, J.-A. E. Månson, C. Löwe, and D. M. Opris, Adv. Funct. Mater. 2010, 20, 3280.

[13] J. S. Bergström, M. C. Boyce, Rubber Chem. Technol. 1999, 72, 633.

[14] M. Kujawski, J. D. Pearse, E. Smela, Carbon 2010, 48, 2409.

[15] A. L. Larsen, P. Sommer-Larsen, O. Hassager, Proc. SPIE 2004, 5385, 108.

[16] S. M. G. Frankær, M. K. Jensen, A. G. Bejenariu, A. L. Skov, Rheol. Acta 2012, 51, 559.

[17] C. Racles, M. Cazacu, B. Fischer, D. M. Opris, Smart Mater. Struct. 2013, 22, 104004.

[18] F. B. Madsen, A. E. Daugaard, S. Hvilsted, M. Y. Benslimane, A. L. Skov, Smart Mater. Struct. 2013, 22, 104002.

[19] F. B. Madsen, I. Dimitrov, A. E. Daugaard, S. Hvilsted, A. L. Skov, Polym. Chem. 2013, 4, 1700. 
[20] B. Kussmaul S. Risse, G. Kofod, R. Waché, M. Wegener, D. N. Mccarthy, H. Krüger, R. Gerhard, Adv. Funct. Mater. 2011, 21, 4589.

[21] A. Egede Daugaard, S. S. Hassouneh, M. Kostrzewska, A. G. Bejenariu, A. L. Skov, Proc. SPIE 2013, 8687, 868729.

[22] L. González, L. Yu, S. Hvilsted, A. L. Skov, RSC Adv. 2014, 4, 36117.

[23] L. Yu, L. B. Gonzalez, S. Hvilsted, A. L. Skov, Proc. SPIE 2014, 9056, 90560C.

[24] P. Zylka, Prz. Elektrotechniczny 2010, 86, 39.

[25] Z. Zhang, S. Sun, L. Liu, K. Yu, Y. Liu, and J. Leng, Proc. SPIE 2009, 7493, 749315.

[26] M. Kujawski, J. Pearse, E. Smela, Proc. SPIE 2010, 7642, 76420R.

[27] G.-H. Chen, D.-J. Wu, W.-G. Weng, W.-L. Yan, J. Appl. Polym. Sci. 2001, 82, 2506.

[28] H. Stoyanov, D. Mc Carthy, M. Kollosche, G. Kofod, Appl. Phys. Lett. 2009, 94, 232905.

[29] M. G. Urdaneta, R. Delille, E. Smela, Adv. Mater. 2007, 19, 2629.

[30] M. Li, Y. G. Jeong, Macromol. Mater. Eng. 2011, 296, 159.

[31] R. Wissert, P. Steurer, S. Schopp, R. Thomann, R. Mülhaupt, Macromol. Mater. Eng. 2010, 295, 1107.

[32] M. Y. Benslimane, H.-E. Kiil, M. J. Tryson, Polym. Int. 2010, 59, 415.

[33] A. L. Larsen, K. Hansen, P. Sommer-Larsen, O. Hassager, A. Bach, S. Ndoni, and M. Jørgensen, Macromolecules 2003, 36, 10063. 
[34] P. Cicuta, I. Hopkinson, Europhys. Lett. 2004, 68, 65.

[35] S. Vudayagiri S. Zakaria, L. Yu, S. S. Hassouneh, M. Benslimane, and A. L. Skov,, Smart Mater. Struct. 2014, 23, 105017.

[36] Z. Shamsul, P. H. F. Morshuis, B. Mohamed, K. V Gernaey, A. Ladegaard, Proc. SPIE 2014, 9056, 90562V.

[37] Masao Doi, Introduction to Polymer Physics, Oxford University Press, New York, 1996.

((Insert Figure here. Note: Please do not combine figure and caption in a textbox or frame.))
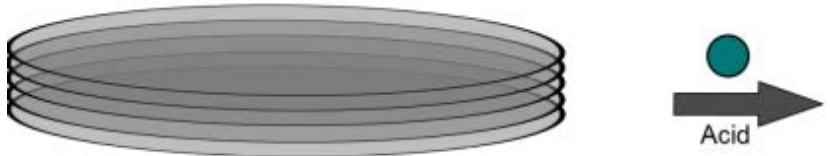

a)

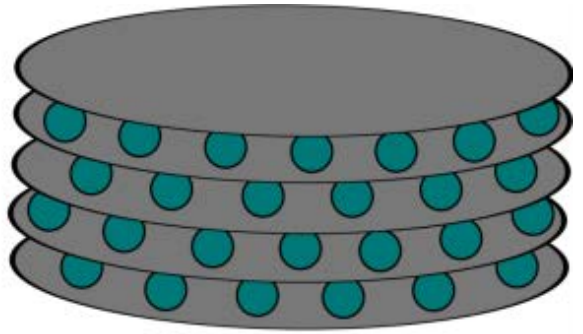

b)

c)




Figure 1. Different types of graphite a) natural graphite, b) expanded graphite and c) exfoliated graphite

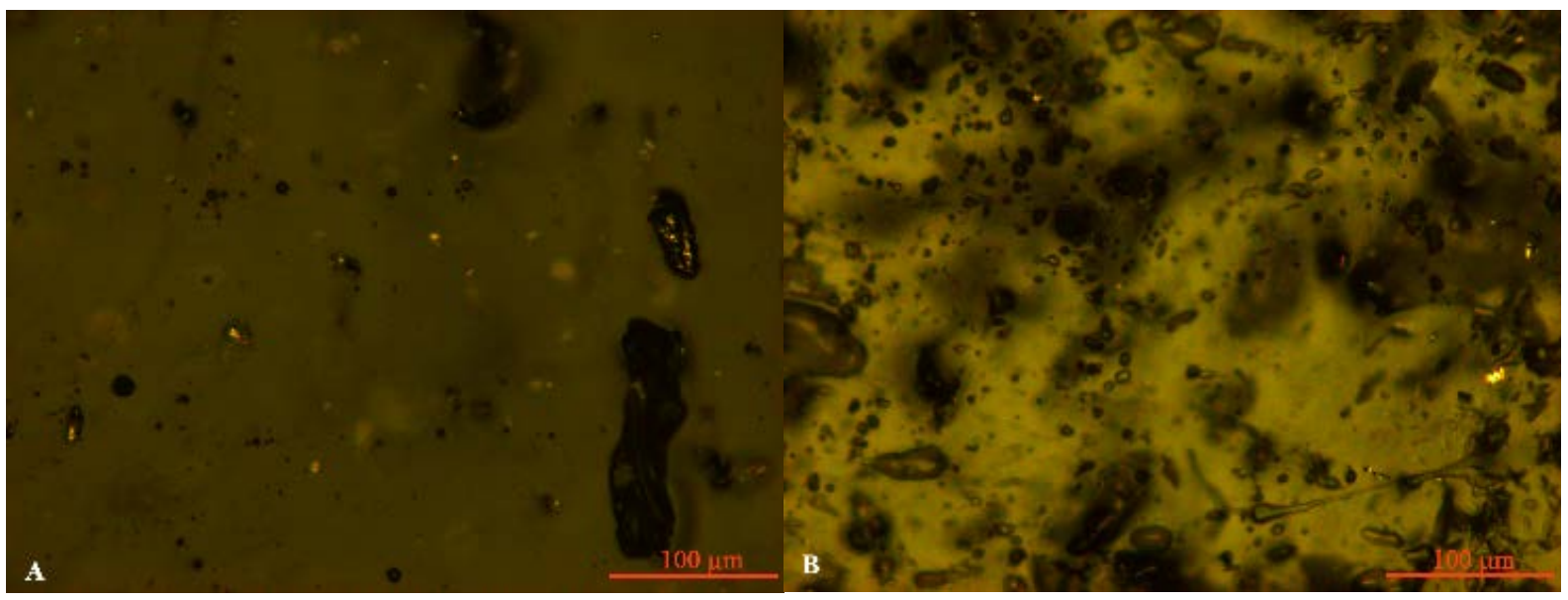

Figure 2. Optical microscope images of the dispersion of EG in a mechanically mixed sample

(A) and a speed mixed sample (B).

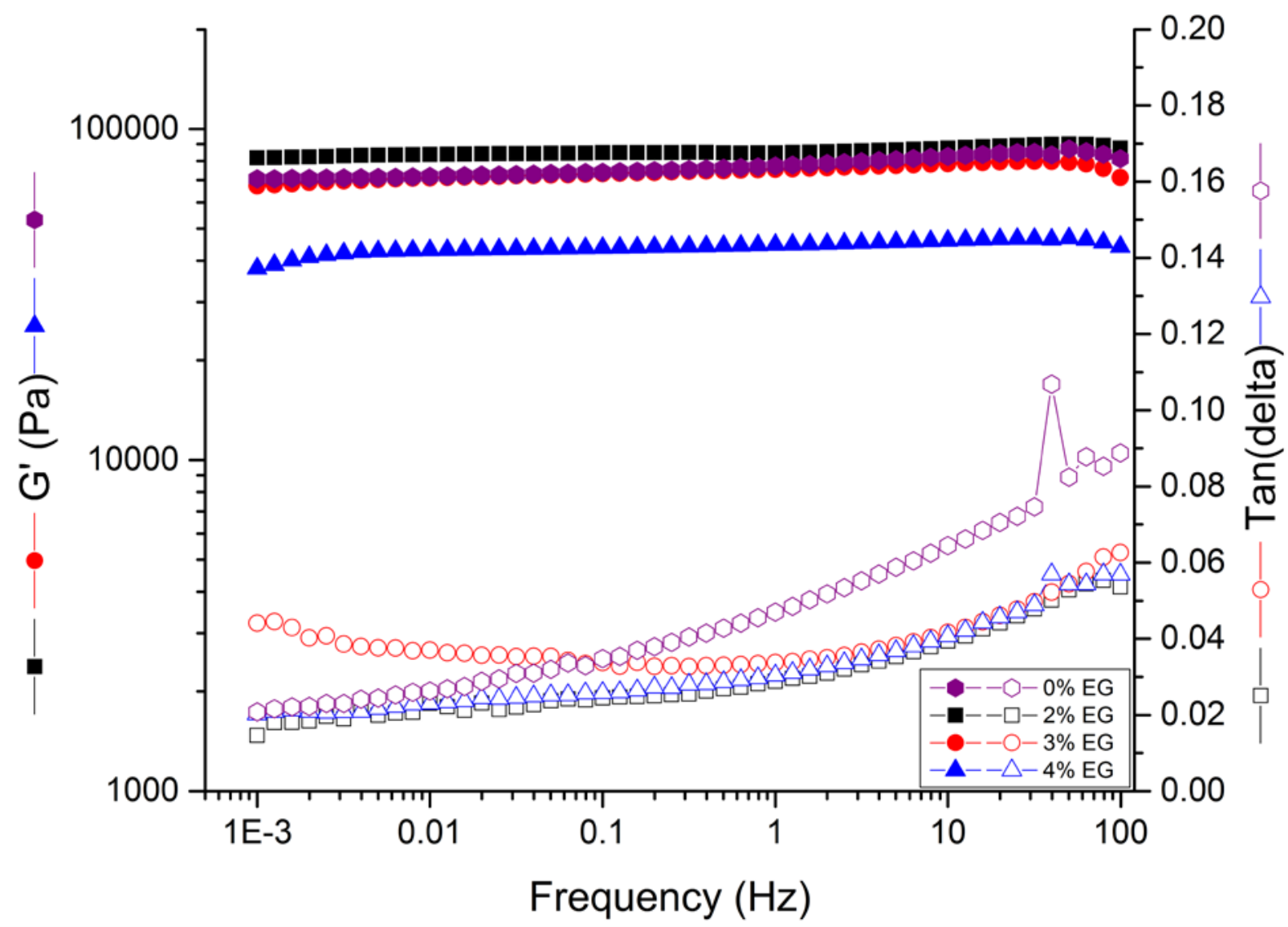


Figure 3. Linear viscoelastic properties of PDMS-V35 network with different loadings of EG compared to the unfilled benchmark elastomer Elastosil RT625.

Table 1. Mechanical properties at room temperature and at $0.01 \mathrm{~Hz}$.

\begin{tabular}{llll}
\hline Sample & $\begin{array}{l}\text { G’ } \\
{[\mathbf{k P a}]}\end{array}$ & $\begin{array}{l}\text { G” } \\
{[\mathbf{k P a}]}\end{array}$ & Tan $\boldsymbol{\delta}$ \\
\hline 2 \% EG & 84 & 1.9 & 0.023 \\
3 \% EG & 71 & 2.6 & 0.037 \\
4 \% EG & 43 & 1 & 0.023 \\
RT625 & 72 & 1.9 & 0.026
\end{tabular}
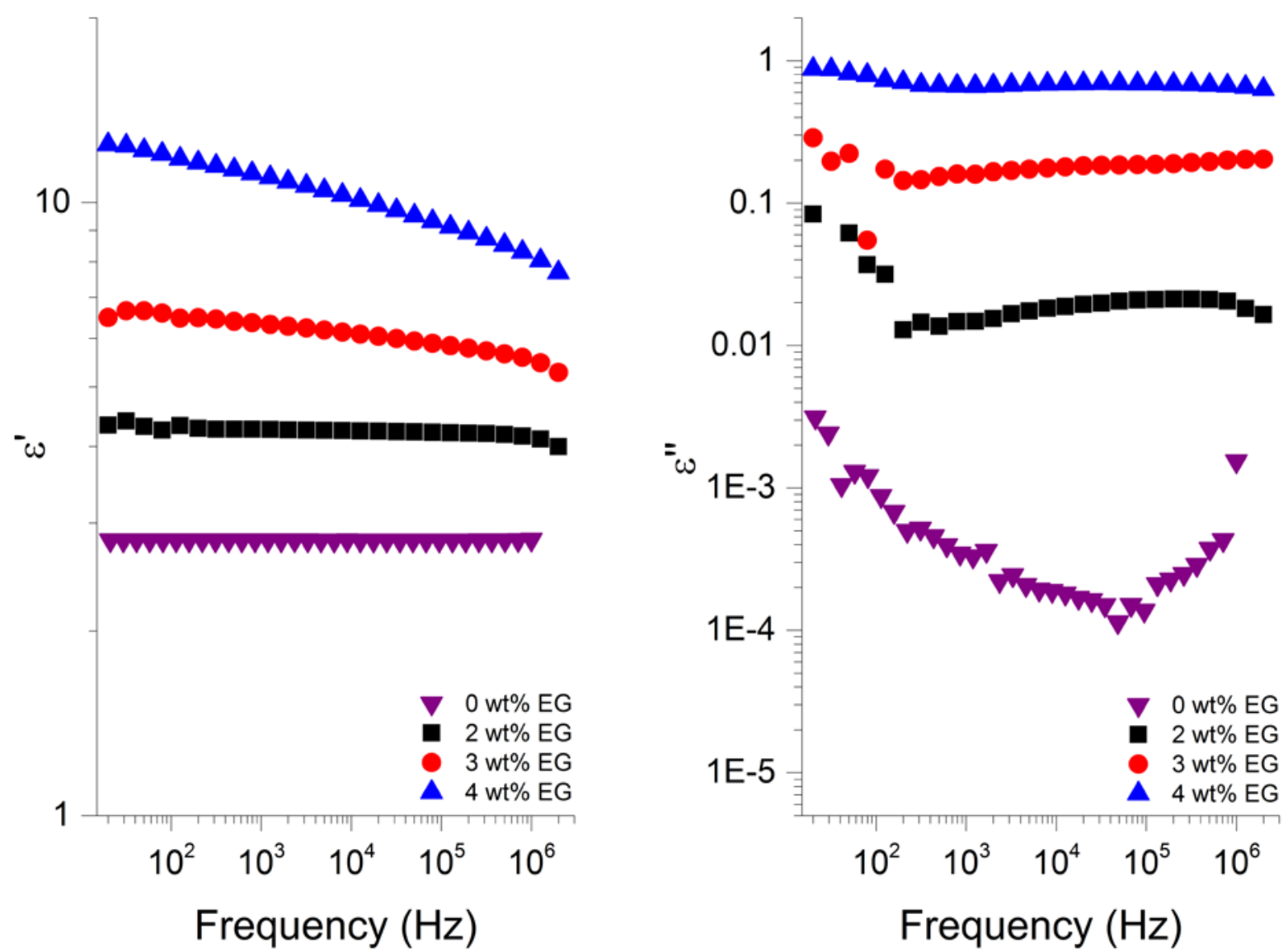

Figure 4. Dielectric permittivity measurements for the different loadings of EG and Elastosil 


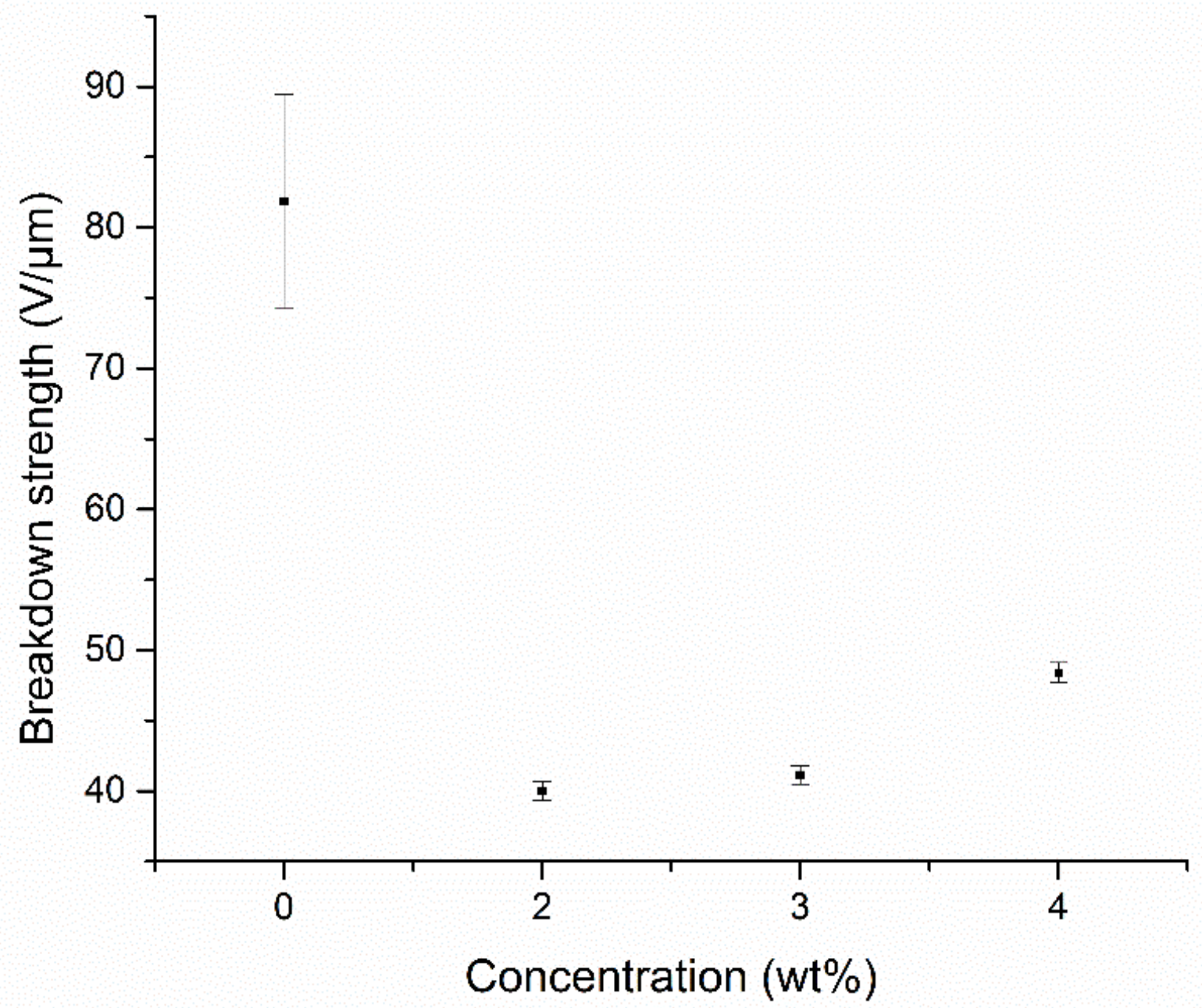

Figure 5. Breakdown strength of the various composites 


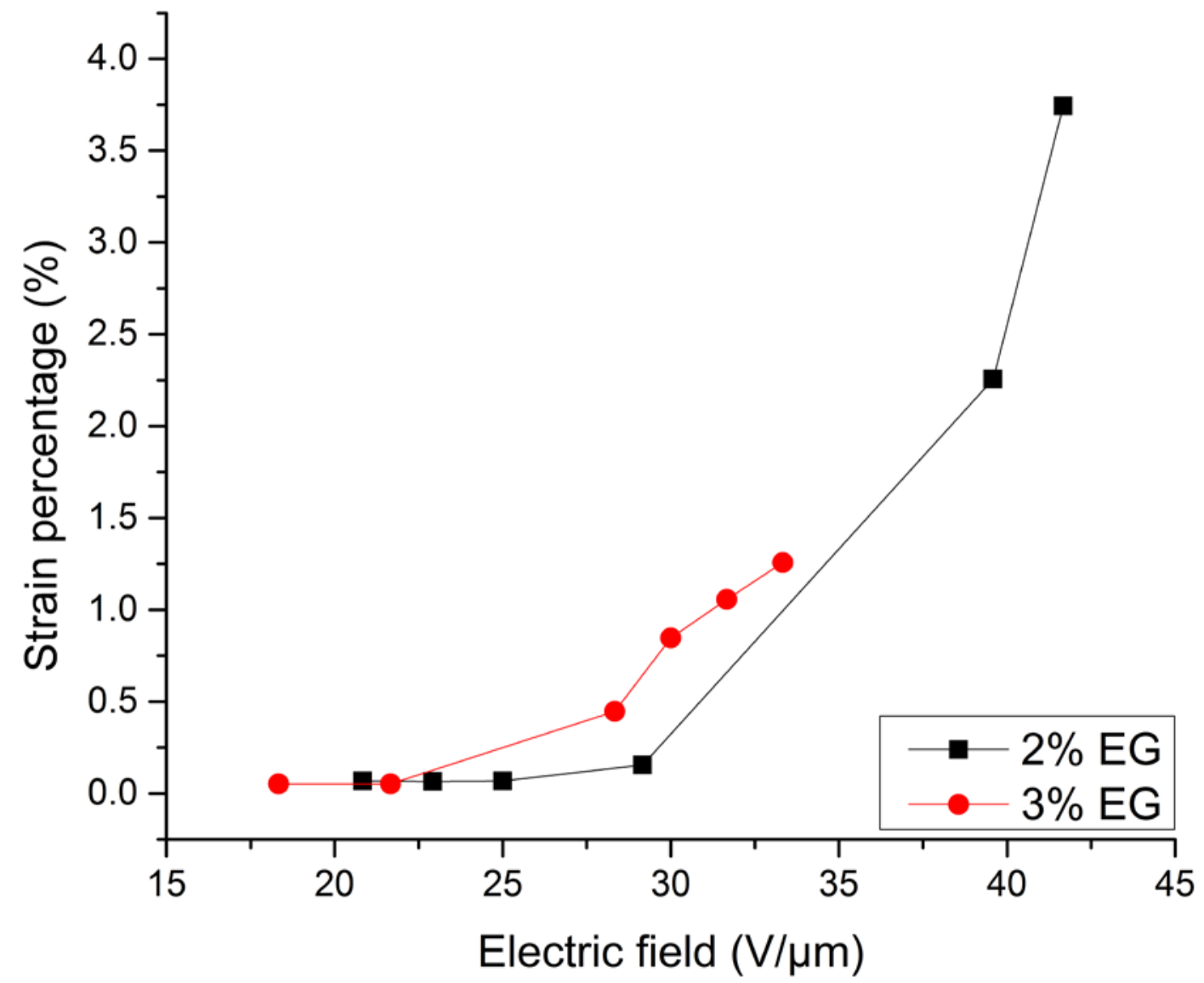

Figure 6. Sample actuation curves for the 2\% and 3\% loadings of EG 

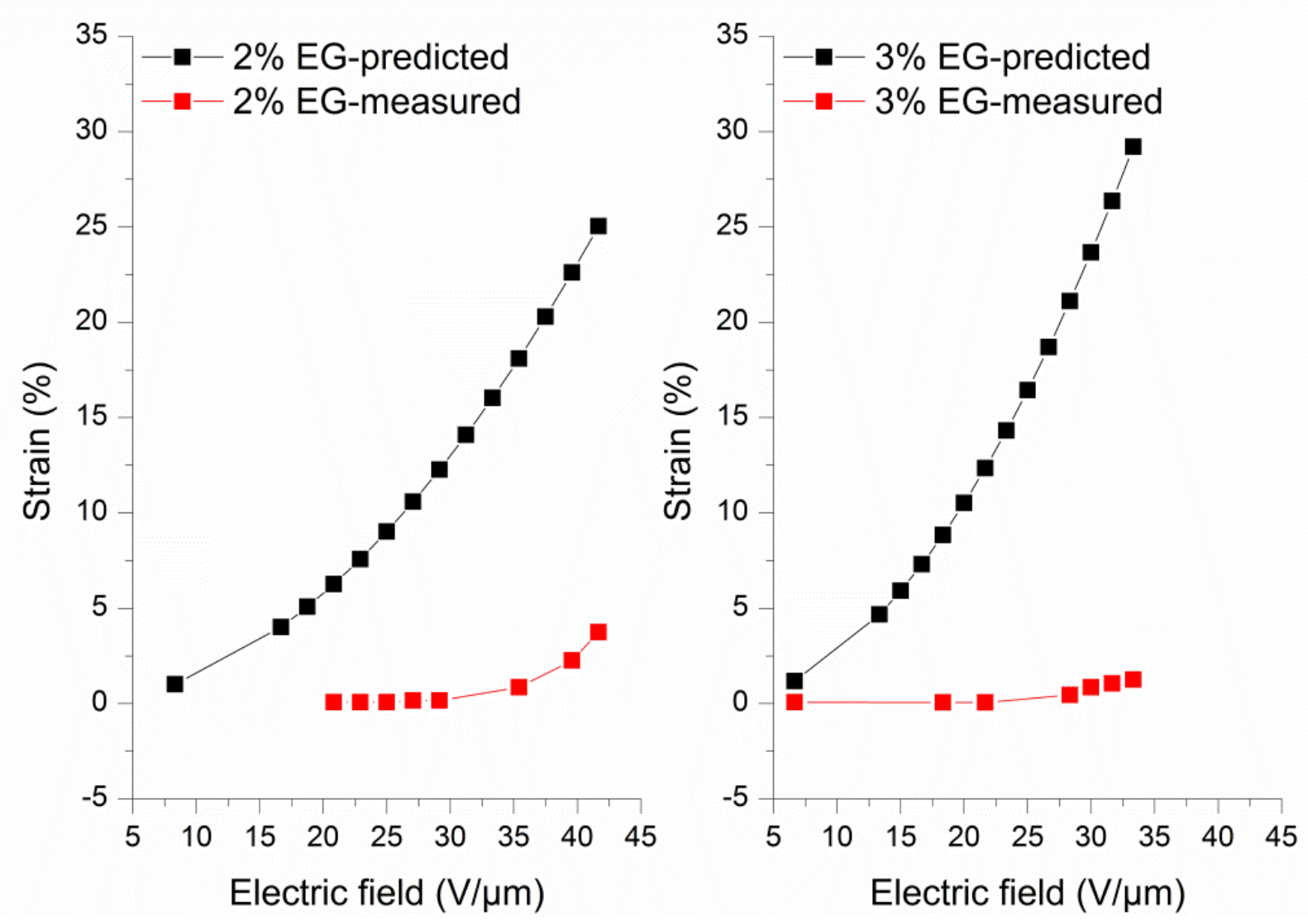

Figure 7. Experimental versus theoretical actuation of composites at room temperature. 